\title{
Cuenca minera de Riotinto (Huelva), paisaje hecho a mano
}

\section{Aquilino Delgado Domínguez ${ }^{a}$}

${ }^{a}$ Museo Minero de Riotinto, Fundación Río Tinto, Plaza del Museo s/n, 21660 Minas de Riotinto. museomineroriotinto@telefonica.net

\begin{abstract}
Resumen
Este trabajo trata del paisaje cultural de la Cuenca Minera de Riotinto. Debido a su amplitud y a la diversidad de elementos que lo componen. Lo hemos zonificado en seis áreas: Zarandas Naya, Filón Norte/San Dionisio, Filón Sur/Masa San Antonio; Mina de Peña de Hierro, elementos arquitectónicos vinculados al patrimonio industrial y diques. Todo este patrimonio generado desde la edad del cobre hasta la actualidad por la actividad minera y metalúrgica, ha dado lugar a un paisaje cultural, continuo y evolutivo.
\end{abstract}

Palabras clave: paisaje cultural, Riotinto, Huelva, Andalucía, España, minería, metalurgia.

\begin{abstract}
This work is about the cultural landscape of the Riotinto Mining Basin. Due to its breadth and the diversity of elements that compose it. We have zoned it into six areas: Zarandas Naya, North Lode/ San Dionisio Lode, South Lode SSan Antonio Lode; Peña de Hierro Mine, architectural elements linked to industrial heritage and dams. All this heritage generated from the copper age to the present by mining and metallurgical activity, has given rise to a cultural, continuous and evolving landscape.
\end{abstract}

Keywords: cultural landscape, Riotinto, Huelva, Andalusia, Spain, mining, metallurgy. 


\section{Introducción. Ubicación y concepto de Paisaje}

La Cuenca Minera de Riotinto se encuentra en la zona central y Este de la provincia de Huelva, limitando al Este con la de Sevilla. Está compuesta por los términos municipales de: Berrocal, El Campillo, Campofrío, La Granada de Riotinto, Minas de Riotinto, Nerva y Zalamea La Real, que ocupan una superficie de $622,71 \mathrm{~km}^{2}$, con una población actual de 17.249 habitantes. La zona central, compuesta por los términos municipales de El Campillo, Minas de Riotinto y Nerva, es donde se encuentra el criadero minero y donde se han desarrollado todas las operaciones mineras y metalúrgicas desde el calcolítico hasta la actualidad (Delgado, 2018). La zona norte, compuesta por los términos municipales de La Granada de Riotinto y Campofrío y la meridional, en el término municipal de Zalamea la Real, se dedican a actividades agrícolas, ganaderas y en menor medida del manejo forestal.

Este trabajo trata sobre el paisaje cultural generado por la actividad minera y metalúrgica en la Cuenca Minera de Riotinto, durante cinco milenios. El concepto de paisaje con el que trabajamos es el recogido en el Convenio Europeo del Paisaje de 20 de octubre de 2000 de Florencia, que lo define en su art. 1 a): "por paisaje se entenderá cualquier parte del territorio tal como la percibe la población, cuyo carácter sea el resultado de la acción y la interacción de factores naturales y/o humanos". Dentro de las categorías de paisaje cultural según la Guía Operativa para la Implementación de la Convención del Patrimonio Mundial está dentro de la categoría de paisaje continuo: "que sigue teniendo un papel social activo en la sociedad contemporánea, conjuntamente con la forma tradicional de vida" dentro de los paisajes evolutivos, entendiendo por estos: “los resultantes de condiciones sociales, económicas, administrativas y lo religiosas, que se han desarrollado conjuntamente y en respuesta a su medio ambiente natural" (Rössler, 2001; 2014).

\section{EI Paisaje Cultural de la Cuenca Minera de Riotinto, desarrollo temático y resultados}

La extensión y diversidad de los bienes patrimoniales que componen el Paisaje Cultural de la Cuenca Minera junto a la disparidad de las características de los mismos (localización, propiedades históricas, técnicas y estado de conservación), nos lleva a estructurar una zonificación del área que nos permite el análisis y estudio de los mismos. Estas zonas (Fig. 1) son: 1) Zarandas-Naya; 2) Filón Norte / San Dionisio; 3 Filón Sur/ Planes /Masa San Antonio; 4) Peña de Hierro; 5) Elementos arquitectónicos vinculados al patrimonio industrial y 6) Diques (Pérez y Delgado, 2009; Delgado y Regalado, 2009; Delgado et al., 2013; Ghislanzoni y Romero, 2014; VVAA, 2016; Delgado, 2018).

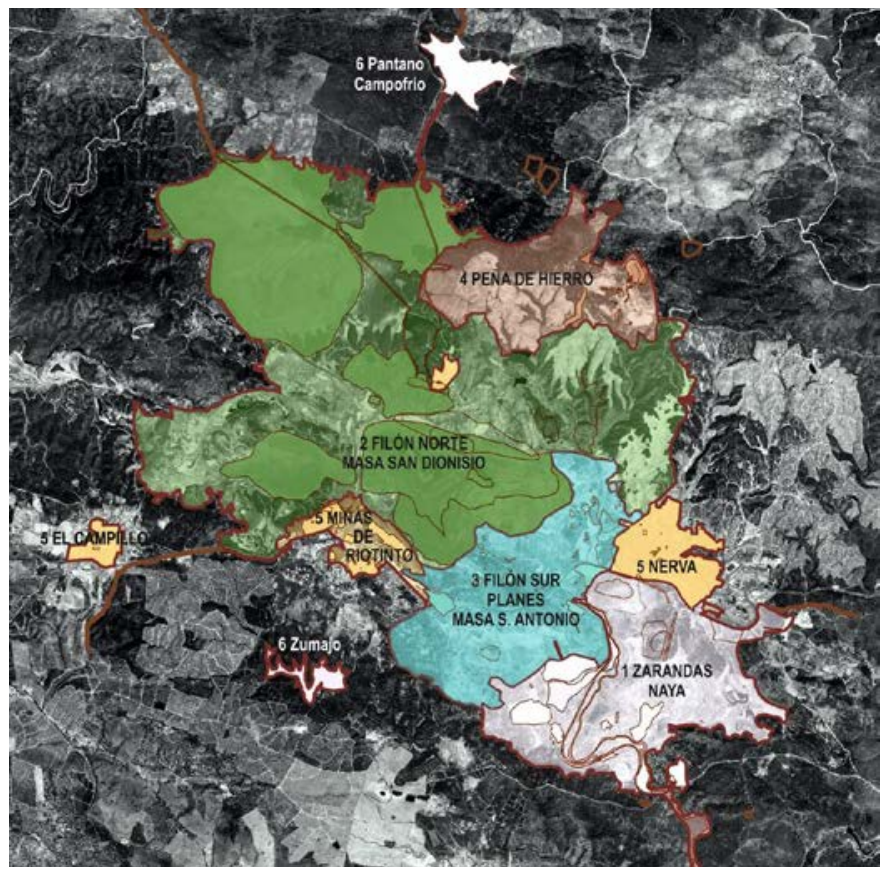

Fuente: Elaboración propia

Fig. 1 Zonificación de la Cuenca Minera de Riotinto 


\subsection{Zarandas-Naya}

Zona situada al Sureste del criadero minero, debe su nombre a la principal actividad que se desarrolló, el tratamiento de mineral y por otro lado al campamento minero de La Naya, que se ubicaba en sus cercanías y donde residían parte de los ferroviarios y los trabajadores de la zona de procesado de mineral. Desde 1901 se concentraron en esta zona todas las operaciones metalúrgicas, primero con la Fundición Bessemer, donde se aplicó el convertidor Bessemer para acero para la obtención de cobre. En 1907 se construyó la Fundición Extensión Bessemer al sur de la anterior, que posteriormente se denominó cómo Fundición Piritas, y donde se llevaron a cabo todas las operaciones metalúrgicas hasta 1970.

En Zarandas también se desarrollaron desde los años 80 del siglo XIX un 30\% de las operaciones hidrometalúrgicas y desde 1926 el 100\%, donde confluyeron todas las aguas procedentes desde la zona de Filón Sur, Cerda, Planes y Marismilla se condujeron mediante canales de madera embreada hasta Zarandas, donde se les unían las de los terreros y las del propio río Tinto (Fig. 2). La planta de cementación para la obtención de cáscara de cobre estuvo operativa, al igual que las balsas para recuperar el sulfato ferroso hasta 1968.

Por último, a fines de los años 50 se instaló en las proximidades de la actual Estación de Zarandas la planta de trituración de piritas provenientes de Corta Atalaya que hasta febrero de 1981 era remolcado por ferrocarril y posteriormente en camiones hasta 1988 cuando cesó la producción, que fue desmontada en 1990.

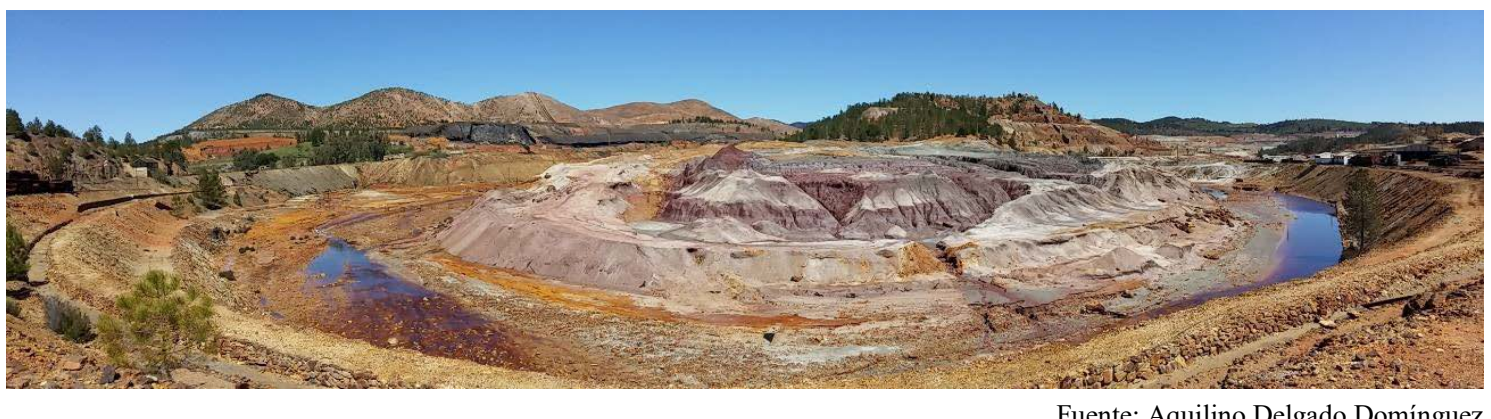

Fuente: Aquilino Delgado Domínguez

Fig. 2 Vista panorámica actual desde el Sur de terreros Naya, por el que discurre el río Tinto. Al norte en la Sierra del Madroñal se ubica la Fundición Piritas y el escorial de la misma. Minas de Riotinto (Huelva)

\subsection{Filón Norte / San Dionisio}

Filón Norte está formado por las masas Dehesa, Lago, Argamasilla, Salomón, Quebrantahuesos, Cerro Colorado y Mal Año. Estas masas han estado explotadas desde época tartésica, con dos grandes períodos de explotación época romana (s. II a.C. - V d.C.) para la obtención de plata y época británica (1873 - 1954) para mineral de cobre y piritas. En 1968 se inició la operación en Cerro Colorado para la obtención de minerales cobrizos y de gossan, mineralización con contenidos atípicos de oro y plata. Para tratar ambas menas se construyó una Unidad Industrial donde se obtenía concentrado de cobre y la planta de oro para obtener este metal y plata mediante el proceso Merrill-Crowe. La bajada de los precios del cobre por debajo del break-even de Riotinto determinó el cierre de la explotación en 2001 . En 2015 el alza de los altos precios del cobre hizo viable la reapertura de Riotinto, por parte de la compañía Emed Tartessus, actualmente Atalaya Mining, desarrollándose desde entonces las operaciones mineras sobre minerales cobrizos, con una ley media de 0,45 y una relación estéril mineral de 1.9:1 (Delgado, 2018).

En esta zona también se ubica Corta Atalaya (Fig. 3) que es la explotación a roza abierta de Masa San Dionisio, es la mina a cielo abierto más grande de Europa. Tiene forma elipsoidal con un eje mayor de $1234 \mathrm{~m}$, y un eje menor de 954 m, una profundidad de $335 \mathrm{~m}$, (piso 23) de los que actualmente unos 95 están cubiertos por el agua. La pendiente en el talud Sur es de $37^{\circ}$ mientras que la del talud Norte es de $42^{\circ}$. La explotación de Corta Atalaya se inició en 1907 y finalizó en 1986, estando en mantenimiento hasta 1992 durante su período de actividad se extrajeron 90 Mt, de material entre mineral y estéril (Delgado et al., 2013). 


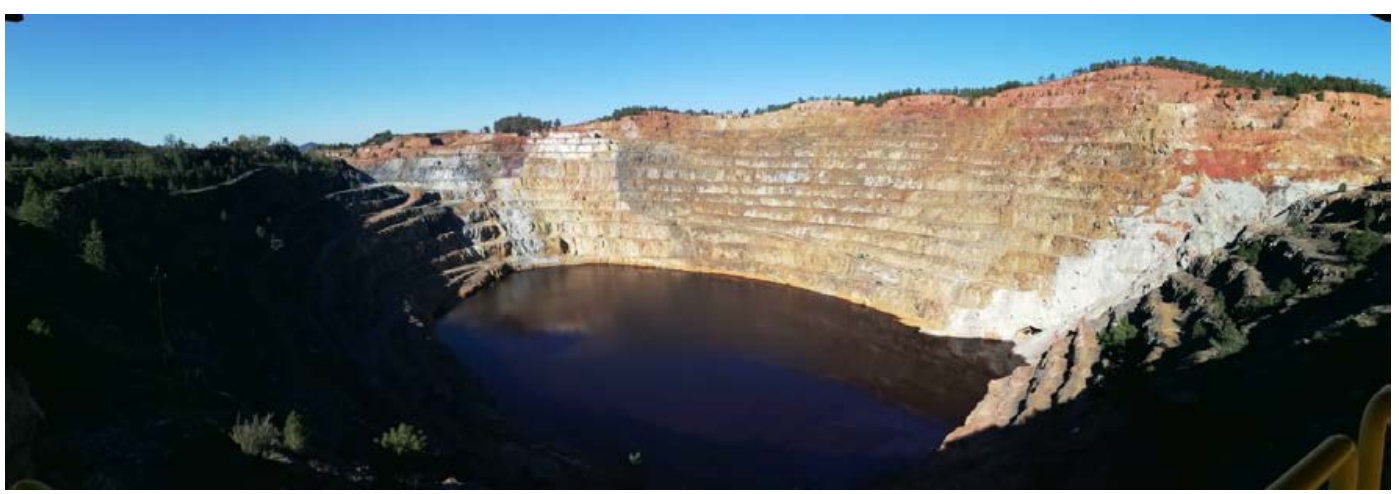

Fuente: Aquilino Delgado Domínguez

Fig. 3 Vista panorámica desde el SE de Corta Atalaya, 2018, Minas de Riotinto / El Campillo (Huelva)

\subsection{Filón Sur / Planes / Masa San Antonio}

En esta zona es donde se desarrollaron el $80 \%$ de las operaciones mineras para la extracción de cobre en época romana y el $90 \%$ de las operaciones mineras entre 1725 y 1890, que se desarrollaron en Masa Filón Sur o Nerva, explotada a cielo abierto entre 1874 y 1952, que fue cubierta por la escombrera de estéril en 1985.

Además de Filón Sur, la tercera zonificación incluye Masa Planes, que estuvo operativa entre 1904 y 1954 y Masa San Antonio, que se preparó para ser explotada por el Pozo Rotilio la caída del precio de los metales hizo que en 1980 se abandonara.

Esta área acogió todas las operaciones metalúrgicas entre 1725 y 1901 con las fundiciones de San Luis, Mina, Los Planes, Desamparados o Huerta Romana (Salkield, 1987) y las operaciones mineralúrgicas entre 1725 y 1916 , cementaciones Nerva, Station, New Station, Marismilla, Planes y Cerda (Pinedo, 1963; Salkield, 1987)

En esta área se desarrollaron los trabajos para la obtención de cobre por vía húmeda artificial, para lo que fue necesaria la de calcinación en "teleras" (Pinedo, 1963; Salkield, 1987) de los minerales cobrizos antes de someterlos al canaleo donde el mineral calcinado se introducía en los pilones donde se introducía agua ácida, con lo que se aumentaba la cantidad de cobre disuelto en el agua que posteriormente se recuperaba precipitándolo con chatarra sin estaño. Este sistema se iniciado en 1798 estará en uso hasta 1908, cuando la puesta en operación de la Fundición Piritas hizo que las teleras no fueran ya necesarias.

Por último, en este sector se ubicaba la salida del túnel 11 y el inicio de la vía general o main line, que unía Riotinto con Huelva y los ramales provenientes de los distintos filones con las zonas donde se procesaba y desde las zonas de trituración con las fundiciones. Esto determinó que hasta el primer tercio del siglo XX se construyera en esta zona la playa de vías más grande de Europa con 42 vías paralelas, con todos los sistemas de cambio, control y cruzamiento necesarios para el tráfico ferroviario. Junto a la playa de vías se construyeron los talleres ferroviarios y las cocheras (Delgado, 2018)

Esta zona desde 1993 y con la reapertura de las operaciones mineras en 2015 ha sido empleada como escombrera minera, lo cual ha tapado parte de esta zona, generando un nuevo paisaje cultural (Fig. 4).

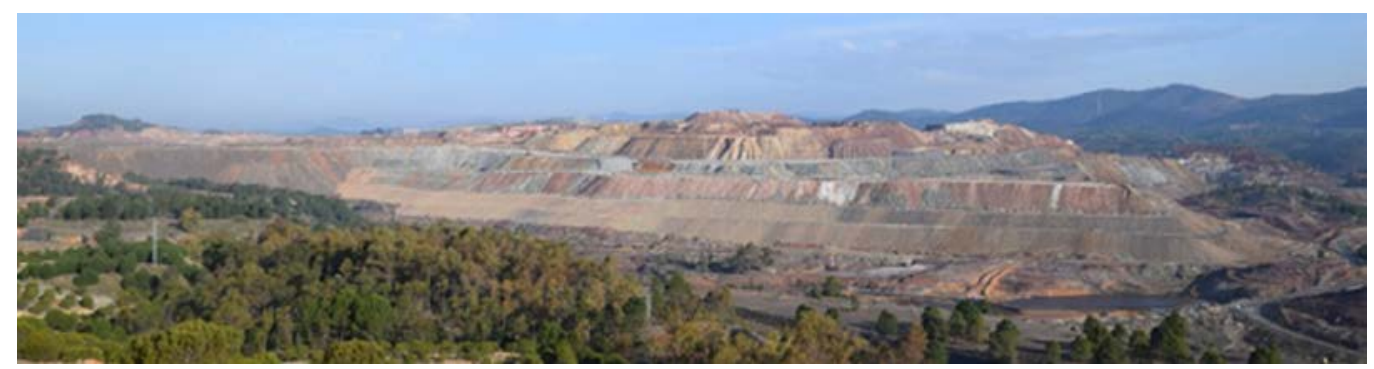

Fuente: Aquilino Delgado Domínguez

Fig. 4 Vista panorámica desde el Sur de la Escombrera Sur (Minas de Riotinto, Huelva) 


\subsection{Peña de Hierro}

Peña de Hierro, está situada al Norte del término municipal de la villa de Nerva (Huelva) a tres kilómetros del núcleo urbano, en la falda Sur de la Sierra del Padre Caro. La primera etapa de explotación minera probada de este grupo minero corresponde a época romana, como evidencia la presencia de galerías romanas. Las operaciones mineras modernas se iniciaron en 1853 por parte de Agapito Artaloitia, que al final de ese año vendió la mina a la compañía minera "Nuestra Sra. de los Reyes" que la tuvo en operación hasta 1866 (Pinedo, 1963). En 1883 se retomaron los trabajos de extracción por una compañía inglesa The Peninsular Copper Company hasta 1900 cuando se disolvió y liquidó por hipotecas. Un año después se constituyó The Peña Copper Mines Limited, empresa británica que trabajó Peña de Hierro durante 54 años, siendo este período el de máxima explotación de este criadero mineral, siendo en este período cuando se desarrollaron al máximo las instalaciones mineras (Delgado y Regalado, 2009).

En 1955 Peña de Hierro pasó a capital español siendo explotada por la Compañía Nacional de Piritas S.A. (CONASA) hasta 1960, cuando se paralizó la extracción por la falta de rentabilidad (Pinedo, 1963). En 1972 pasó la titularidad a Río Tinto Patiño, cesando en ese mismo año los trabajos de mantenimiento que se venían desarrollando desde hacía más de diez años. Desde ese momento Peña de Hierro estuvo abandonada convirtiéndose en fuente de materiales constructivos (Delgado y Regalado, 2009). En 1987, con la creación de la Fundación Río Tinto, Peña de Hierro pasó a formar parte de sus activos, desde mediados de los noventa se ha venido trabajando en su rehabilitación patrimonial de distintos bienes patrimoniales (Corta, Túnel Sta. María, Castillete, Casa de Máquinas, trituradora, etc.) (Fig. 5) (Delgado y Regalado, 2012).

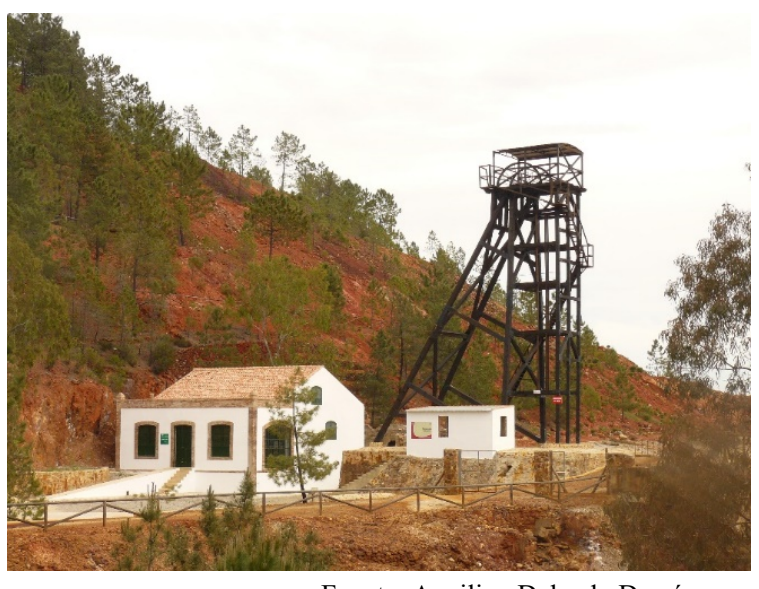

Fuente: Aquilino Delgado Domínguez

Fig. 5 Castillete del Pozo Maestro y su Casa de máquinas rehabilitadas

\subsection{Elementos arquitectónicos vinculados al patrimonio industrial}

Además de las infraestructuras necesarias para las distintas operaciones mineras en las distintas poblaciones y aldeas las distintas compañías mineras construyeron distintos edificios para cubrir las necesidades de los trabajadores y sus familias (escuelas, viviendas, capillas, iglesias, dispensarios médicos o economatos) (Fig. 1). Aunque creemos interesante diferenciar Minas de Riotinto, del resto de las poblaciones, pues todo el término municipal y el casco urbano fue propiedad de la Compañía desde 1873. La población original, Río Tinto, empezó a ser fagocitada desde el principio del siglo XX por el avance de corta de Filón Sur, naciendo en el Valle el actual Minas de Riotinto que fue creciendo hasta conformar el pueblo actual. Siendo costeado por las distintas compañías mineras todas las edificaciones (Iglesia, escuelas, plaza de abastos, piscinas, hospital, almacenes y viviendas).

En el núcleo urbano de Nerva hay menos presencia de edificios industriales construidos por la compañía o propiciados por ella, como la Estación y la Factoría, otros equipamientos destinados a dar servicio al personal de la Compañía o sus familias, como el Convento de la Milagrosa, escuela para hijas de los obreros, economato e incluso participó en la construcción de edificios civiles como el cementerio municipal. 
Los elementos arquitectónicos vinculados al Patrimonio Industrial en el núcleo de Zalamea La Real son aquellos relacionados con el ferrocarril, pues por esa población pasaban dos líneas férreas, el Fc. del Buitrón y el Fc. Minero de Río Tinto. En Berrocal sucede lo mismo, los edificios industriales son aquellos vinculados al Fc. Minero de Río Tinto, como la Estación de Berrocal, la casa de señales y las viviendas de los factores.

En el Campillo, el pueblo más joven de la Cuenca Minera de Riotinto, pues se independizó de Zalamea La Real en 1931, las construcciones vinculadas al patrimonio industrial, al igual que en las dos poblaciones anteriores son aquellas asociadas al ferrocarril, cómo la Estación o la Factoría, junto con el grupo de viviendas que construyó Rio Tinto Patiño a principios de los setenta, denominado popularmente como "El Campillo Nuevo" (Delgado, 2018).

\subsection{Diques}

Por último, otras infraestructuras asociadas al Patrimonio Industrial son los diques, las compañías mineras que trabajaron en la Cuenca Minera de Riotinto, necesitaron disponer de agua, además de para los procesos industriales para abastecer de agua potable a la población (Fig. 1). En 1881 Río Tinto Co. Ltd. construyó el dique de Campofrío en el término municipal del mismo nombre, que dotó de agua potable las fuentes públicas y lavaderos, a partir de la primera década del siglo XX a las viviendas. El aumento de consumo de agua a mediados de los años 50 del siglo XX determinó entre 1955 y 1957 que se ampliara de nuevo, estando actualmente en uso. Río Tinto Co. Ltd. construyó entre 1907 y 1908 otro dique en el actual término municipal de El Campillo, el Zumajo que sirvió para dotar de agua al Departamento de Tierras y Ganado de la propia compañía minera, tener otra reserva de agua potable para consumo de boca, este dique fue empleado por parte de los directivos para la práctica de deportes náuticos, actualmente sigue en uso para el riego de frutales por parte de Rio Tinto Fruit. En Nerva, The Peña Copper Mines, construyó dos pantanos, Tumbanales 1 y 2, para uso industrial, al igual que el Zumajo se emplean en la actualidad para el riego de frutales (Delgado, 2018).

\section{Conclusión}

Como conclusión todos los bienes patrimoniales generados entre el calcolítico y la actualidad, con distinto grado de conservación son el resultado de 5.000 años de actividad minera y metalúrgica, que conjugados han conformado y dado lugar a un paisaje cultural, continuo y evolutivo, como ya se ha citado, pues las operaciones mineras actuales crean diariamente un nuevo paisaje minero.

La importancia patrimonial de la zona que nos ocupa determinó que la Junta de Andalucía, entidad que tiene transferidas las competencias en materia cultural, la declarara Bien de Interés Cultural, con la categoría de Zona Patrimonial (Decreto 504/2011 de 16 de octubre). Además, la Cuenca Minera de Riotinto, alberga un ecosistema único en el mundo, el río Tinto, que fue declarado Paisaje Natural Protegido (Decreto 558/2004, de 14 de diciembre), por último, la montera de gossan de Peña de Hierro, fue declarada Monumento Natural de Andalucía (Decreto 48/2010 de 3 de marzo).

Además de todo lo expuesto la Cuenca Minera de Riotinto cumple varios criterios de la Convención de Patrimonio Mundial de 1972: (ii) pues en esta zona se atestigua el intercambio de valores humanos, desde el calcolítico hasta la actualidad; el (iv) la Cuenca Minera conforma un paisaje antropizado fruto de cinco milenio de actividad minera y metalúrgica, con distinto grado de conservación en el territorio dispone de todos los elementos significativos de la actividad ya mencionada, permitiendo explicar su evolución de forma diacrónica y sincrónica; (v) la zona que nos ocupa es uno de los ejemplos más representativos a nivel mundial de la interacción del hombre con la extracción de minerales y la obtención de metal, dando lugar a un paisaje cultural que se transformó con cada período de explotación; (vii y viii) El paisaje de la Cuenca Minera de Riotinto, con sus colores, orografía e incluso los olores es minero y único en el mundo en cuanto a sus dimensiones y sincronía y por último el (ix) el área de estudio cuenta con un ecosistema único en el mundo, el río Tinto, además de contar con una especie endémica la erica andevalensis o brezo de las minas. Todo lo expuesto demuestra que la Cuenca Minera de Riotinto cuenta con todos los requisitos para ser declarado Patrimonio Mundial. 


\section{Referencias}

Delgado Domínguez, A., y Regalado Ortega, M. C. (2009). La Rehabilitación patrimonial de la mina de Peña de Hierro (Nerva, Huelva), Parque Minero de Riotinto (Huelva, España). De Re Metallica, 12, 55-63.

Delgado Domínguez, A., y Regalado Ortega, M. C. (2012). Catálogo del Patrimonio Minero Industrial de la Mina de Peña de Hierro (Nerva, Huelva - España). De Re Metallica, 18, 13-27.

Delgado Domínguez, A., Rivera Jiménez, T., Pérez Macías, J. A., y Regalado Ortega, M. C. (2013). La catalogación del Patrimonio Minero Industrial de la Cuenca Minera de Riotinto mediante la aplicación del sistema de información geográfico (SIG). De Re Metallica, 20, 83-95.

Delgado Domínguez, A. (2018). Patrimonio Industrial de la Faja Pirítica Ibérica, Minas de Riotinto. En J. Suárez y N. Raposo (Eds.), Jornadas de Arqueología, Historia y Minería de la Faja Pirítica Ibérica (pp. 55-84). Puebla de Guzmán: Asociación Herrerías.

Ghislanzoni, M., y Romero Dacal, M. (2014). La Zona Patrimonial de la Cuenca Minera de Riotinto-Nerva, como modelo de protección de un paisaje cultural. En VII Congreso Internacional de Ordenación del Territorio (pp. 1-15). Madrid: Asociación Interprofesional de Ordenación del Territorio.

Pérez Macías, J. A., y Delgado Domínguez, A. (2009). Elaboración de la Documentación Técnica sobre el Patrimonio Industrial Minero del Sitio Histórico de Riotinto, $n^{\circ}$ expte. B09059V21HU. Consejería de Cultura de la Junta de Andalucía, Delegación Provincial de Huelva, (inédito).

Pinedo Vara, I. (1963). Piritas de Huelva, su historia, minería y aprovechamiento. Madrid: Editorial Summa.

Rössler, M. (2001). Los paisajes culturales y la Convención del Patrimonio mundial cultural y natural: resultados de reuniones temáticas previas. En E. Múgica Barreda (Ed.), Paisajes culturales en los Andes. Memoria narrativa, Casos de estudio, Conclusiones y Recomendaciones de la Reunión de Expertos, Arequipa y Chivay, Perú, 17-20 de mayo 1998, (pp. 47-55). Lima: UNESCO.

Rössler, M. (2014). World Heritage Cultural Landscapes: 1992-2012. En Conserving Cultural Landscapes (pp. 47-64). Londres: Routledge.

Salkield, L. U. (1987). A Technical history of the Rio Tinto mines: some notes on exploitation from pre-phoenician times to the 1950s. Londres: Institute of Mining and Metallurgy.

V.V.A.A. (2016). Paisaje Minero de Riotinto (Huelva). Sevilla: Junta de Andalucía. 